\title{
Reação de cultivares e linhagens de feijoeiro em relação a Meloidogyne javanica e Fusarium oxysporum f. sp. phaseoli
}

\author{
Reaction of cultivars and lines of bean in relation to Meloidogyne javanica and \\ Fusarium oxysporum f. sp. phaseoli
}

\section{Gervásio Simão $^{\mathrm{I}}$ Idenize Pedrina Orsini $^{*}$ Ciro Hideki Sumida $^{\mathrm{I}}$ Martin Homechin (in memoriam) $^{\mathrm{I}}$ Débora Cristina Santiago' ${ }^{I}$ Vânia Moda Cirino ${ }^{\text {II }}$}

RESUMO

Os nematoides do gênero Meloidogyne e o fungo do gênero Fusarium sp. podem limitar a produção das culturas. Assim, no presente trabalho, o objetivo foi avaliar o comportamento de nove cultivares e duas linhagens de feijoeiro $\boldsymbol{P}$. vulgaris ao nematoide $\boldsymbol{M}$. javanica $e$ ao fungo $\boldsymbol{F}$. oxysporum f. sp. phaseoli (Fofsp) e a interação destes. Os ensaios foram conduzidos sob condições de casa-de-vegetação, em delineamento estatístico inteiramente ao acaso, com quatro tratamentos com oito repetições na avaliação efetuada aos 35 dias e quatro tratamentos para a avaliação aos 60 dias da inoculação. Na primeira avaliação, foram determinados o número de galhas (NG) para M. javanica, no sistema radicular, e a severidade (SEV), para F. oxysporum f. sp. phaseoli. $\mathrm{Na}$ avaliação aos 60 dias, as análises foram idênticas aos da primeira avaliação, porém, determinou-se também o fator de reprodução do nematoide. Nas duas avaliações realizadas, foi observado que M. javanica isoladamente ou em associação com $\boldsymbol{F}$. oxysporum foi parasita de todas as linhagens e cultivares de feijão testadas. Para F. oxysporum isoladamente, as cultivares 'Pérola' e 'IAPAR 14'e a linhagem LP99-85 comportaram-se como resistentes. Na cultivar 'Pérola', observou-se um aumento significativo da severidade dos sintomas à murcha de Fusarium, quando o fungo estava associado a M. javanica.

Palavras-chave: murcha de Fusarium, nematoides de galhas, Phaseolus vulgaris, resistência varietal.

\section{ABSTRACT}

The nematode of the genera Meloidogyne and the fungi Fusarium may reduce the crop yield. So, the present paper had as objective to evaluate the behavior of nine cultivars and two lines of bean P. vulgaris to the nematode M. javanica the fungi F. oxysporum $f$. sp. phaseoli (Fofsp) and its interaction.
The trials were conducted under greenhouse conditions at randomized design using four treatments replicated eight times for the evaluations made at 35 days, and four treatments for the evaluation at 60 days after inoculation. The first evaluation was determined the number of galls (NG) for M. javanica root system, and severity (SEV) for F. oxysporum f. sp. phaseoli. In assessing the 60 days, the analysis were identical to the first however, it was determined the factor of nematode reproduction (FR). For both evaluations, it was observed that M. javanica alone or in association with $\mathbf{F}$. oxysporum, were parasite of all tested bean cultivars and lines. For F. oxysporum alone the cultivars 'Perola', 'IAPAR 14' and the line LP99-85, behaved as resistants. For the cultivar 'Perola', it was observed a significant increase of severity of the symptom of Fusarium when the fungi associated with M. javanica.

Key words: Fusarium wilt, Phaseolus vulgaris, reaction variety, root knot nematode.

\section{INTRODUÇÃO}

No Brasil, os nematoides formadores de galhas, em especial Meloidogyne javanica (TREUB) CHITWOOD, e fungos do solo, como Fusarium oxysporum f. sp. phaseoli KENDRICK \& SNYDER, podem ser responsabilizados pela baixa produtividade e pelas perdas da cultura do feijoeiro (Phaseolus vulgaris L.). Essas perdas decorrem principalmente em regiões com elevadas temperaturas, fator responsável pela predisposição, interferindo na resistência das plantas ao parasitismo por esses patógenos (RIBEIRO \& FERRAZ, 1983).

IDepartamento de Agronomia, Centro de Ciências Agrárias, Universidade Estadual de Londrina (UEL), CP 6001, 86055-990, Londrina, PR, Brasil. E-mail: idenizeorsini@yahoo.com.br. *Autor para correspondência.

IIInstituto Agronômico do Paraná (IAPAR), Londrina, PR, Brasil. 
O feijoeiro é considerado bom hospedeiro de nematoides do gênero Meloidogyne, principalmente M. incognita (KOFOID \& WRITE) e M. javanica (VIEIRA, 1993). As perdas decorrentes da presença desses nematoides no solo podem atingir $90 \%$ (AGUDELO, 1980). Fontes de resistência ao gênero Meloidogyne em feijoeiro têm sido detectadas, porém pouco exploradas (CARNEIRO \& FERRAZ, 1992; ROBERTS, 1992). No Brasil, até o presente momento, não têm sido encontradas fontes de resistência com elevada eficiência dentro do gênero Phaseolus, mas sim cultivares com resistência moderada, sem capacidade para reduzirem satisfatoriamente a reprodução do parasita (MOURA \& REGIS, 1987; PEDROSA et al., 2000). WALBER et. al. (2003), ao avaliarem acessos de feijoeiro "Bambuí”, “Iapar 57” e "Rio Doce”, observaram que estes são possuidores de resistência múltipla a todas as espécies e raças dos nematoides de galhas, indicando a possibilidade do aproveitamento desses acessos como possíveis fontes de resistência, para emprego em programas de melhoramento para resistência.

Ao lado dos nematoides, agentes causais de doenças, o fungo $\boldsymbol{F}$. oxysporum f. sp. phaseoli também é importante agente causal de murcha no feijoeiro. Ele apresenta diferentes raças fisiológicas, com alteração do comportamento das cultivares e linhagens quanto a sua suscetibilidade ou resistência às raças fisiológicas.

Porém, pouco é conhecido sobre a interação entre fungos e nematoides. Um dos primeiros relatos a respeito dessa interação foi realizado por ATKINSON (1892) no Alabama, EUA, que observou maior severidade da murcha de Fusarium em algodoeiro, quando na presença de nematoides do gênero Meloidogyne. Outros estudos, em diferentes culturas, também têm sido relatados, mostrando que a murcha de Fusarium se torna mais severa quando na presença de nematoides (JULIATTI \& RUANO, 1997; RUANO, 1984).

Especificamente para o feijoeiro, estudos da avaliação dos efeitos da interação de nematoides na incidência de doenças fúngicas são escassos até o presente. A literatura cita estudos de BOOKBINDER \& BLOOM (1980), que observaram efeito sinergistico entre $\boldsymbol{M}$. incognita e o parasita obrigatório Uromyces phaseoli. Também há estudos de SINGH et al. (1981), os quais verificaram aumento da murcha de Fusarium sp. em feijoeiro, quando da inoculação simultânea de Fusarium sp. e $\boldsymbol{M}$. javanica.

$\mathrm{O}$ presente trabalho tem como objetivo avaliar a reação de cultivares e linhagens de feijoeiro ao nematoide $\boldsymbol{M}$. javanica e ao fungo $\boldsymbol{F}$. oxysporum $\mathbf{f}$. sp. phaseoli e as interações entre eles, buscando subsidiar a escolha de cultivares pelos produtores e gerar conhecimentos para auxiliar programas de melhoramento genético que visam à obtenção de cultivares tolerantes e/ou com resistência.

\section{MATERIAL E MÉTODOS}

O experimento foi conduzido sob condições de casa-de-vegetação, na Universidade Estadual de Londrina (UEL), Paraná (PR), tendo sido avaliado o comportamento das cultivares: ‘Rubi’, 'IPR Graúna’, 'Xamego', 'IPR Juriti', 'IAPAR 81', 'IAPAR 14', 'Pérola', 'IPR Uirapuru’ e 'IPR Chopim’ e duas linhagens de feijoeiros pertencentes ao programa de melhoramento do IAPAR: LP99-85 e LP98-123, frente ao parasitismo de $\boldsymbol{M}$. javanica e $\boldsymbol{F}$. oxysporum f. sp. phaseoli e interações entre eles. Para a avaliação da reação a $\boldsymbol{M}$. javanica, as plantas foram desenvolvidas em vasos de cerâmica, com capacidade para cinco kg de substrato, mistura de areia + solo na proporção de 2:1, tratado com brometo de metila $\left(150 \mathrm{~g} \mathrm{~m}^{-3}\right.$ ) (quando ainda podia ser utilizado). Em cada vaso, foram semeadas duas sementes, com desbaste no $12^{\circ}$ dia da emergência, deixando-se uma plântula por vaso. O inóculo de $\boldsymbol{M}$. javanica foi obtido a partir da sua multiplicação em plantas de tomateiro (Lycopersicon esculentum L.) da cultivar 'Rutgers', sob condições de casa-devegetação. A extração dos ovos foi realizada seguindose a técnica proposta por HUSSEY \& BARKER (1973), modificada por BONETTI \& FERRAZ (1981), com trituração das raízes do tomateiro durante 30 segundos em liquidificador. As suspensões foram de 1000 ovos e/ou juvenis $\mathrm{ml}^{-1}$. A inoculação foi realizada aos 15 dias da emergência, nas plântulas de feijoeiro em desenvolvimento, por meio da deposição de cinco ml de uma suspensão contendo (5.000 ovos e/ou juvenis planta $^{-1}$ ), em dois orifícios no solo, na região próxima ao colo da planta a uma profundidade de 2 a $3 \mathrm{~cm}$.

Para inoculação do $\boldsymbol{F}$. oxysporum f. sp. phaseoli, o isolado foi obtido do isolamento de raízes de plantas de feijoeiro com sintomas da doença em meio de batata-dextrose-ágar (BDA) acidificado para $\mathrm{pH}$ 4,5-5,0. Em seguida, procedeu-se à multiplicação em meio de BDA, por oito dias, sob luz contínua e temperatura de $27^{\circ} \mathrm{C} \pm 1^{\circ} \mathrm{C}$. A suspensão do inóculo foi obtida a partir da adição de $10 \mathrm{ml}$ de água destilada esterilizada em cada placa sobre as colônias desenvolvidas e remoção dos esporos e micélios com auxilio de pincel de cerdas macias. A suspensão foi ajustada para $10^{6}$ conídios $\mathrm{ml}^{-1}$, por meio de contagem desta em hemacitômetro. 
Na inoculação, as sementes das cultivares e linhagens de feijoeiro foram submetidas à assepsia com hipoclorito de sódio a $2 \%$, por três minutos, e lavadas em água corrente, pré-germinadas em papel de filtro em gerbóx, sob câmara úmida tipo BOD a $28^{\circ} \mathrm{C}$, por quatro dias. Em seguida, foram transplantadas para vasos de barro contendo areia autoclavada e mantidas em condições de laboratório. Aos 15 dias da emergência, procedeu-se à avaliação. Para a inoculação, as plântulas foram removidas dos vasos, suas raízes foram lavadas em água corrente e 1/3 do total das raízes foi eliminado, sendo o remanescente mergulhado em $10 \mathrm{ml}$ da suspensão de inóculo contendo $10^{6}$ esporos $\mathrm{ml}^{-1}$ por $10 \mathrm{~m}$, segundo metodologia de COSTA et al. (1989). Estas foram novamente plantadas nos vasos contendo substrato 1:1 de areia e solo e estes foram mantidos até a avaliação, sob condições de casa-de-vegetação.

Para a avaliação da interação entre nematoide e fungo, a inoculação dos dois patógenos foi simultânea no $15^{\circ}$ dia da emergência. As combinações da inoculação dos patógenos foram: Fusarium, Fusarium + M. javanica, M. javanica e a testemunha sem inoculação (apenas água destilada), totalizando quatro tratamentos. As avaliações foram realizadas aos 35 e 60 dias da inoculação. O delineamento experimental foi o inteiramente casualizado composto por 11 genótipos, com quatro tratamentos, inoculados artificialmente. Foram utilizadas oito repetições para os tratamentos da primeira época e quatro para a segunda, e cada parcela foi constituída por um vaso contendo uma planta. Na primeira avaliação, determinou-se o número de galhas (NG) por sistema radicular por planta e, para $\boldsymbol{F}$. oxysporum f. sp. Phaseoli, foram determinados a severidade (SEV) da doença pelos sintomas internos e externos ao sistema vascular. Na segunda avaliação, foi determinado o fator de reprodução (população final/população inicial), seguindo-se a escala proposta por OOSTENBRINK (1966), em que: $0=\mathrm{I}$ (imune); menor que 1,0=T (tolerante); maior que $1,0=\mathrm{S}$ (suscetível). Para tanto, procedeu-se à extração dos nematoides das raízes e dos demais procedimentos seguindo-se a técnica de BONETTI \& FERRAZ (1981). Na avaliação para Fusarium, aos 35 e 60 da inoculação, adotou-se a escala de notas adaptada de CRUZ et al. (1974), sendo 0 - ausência de sintomas; 1 - ausência de sintomas externos de murcha e presença de escurecimento vascular confinado à raiz principal; 2 - sintomas externos iniciais da doença (clorose e murcha) e escurecimento vascular, atingindo o terço inferior do caule; 3 - sintomas bem definidos da doença (clorose, murcha, lesão foliar e seca das folhas); 4 sintomas bem definidos da doença e escurecimento atingindo o terço superior da planta ou as plantas mortas, com base na observação dos sintomas externos visíveis na parte aérea e internos na planta, por meio de corte e observação de alteração do sistema vascular.

Os dados foram submetidos ao teste de normalidade de Shapiro-Wilk, indicando distribuição normal, e a homogeneidade das variâncias foi testada utilizando Hartley. As análises estatísticas dos dados obtidos foram efetuadas utilizando-se o programa SAS (1990). Para a variável, fator de reprodução (FR), efetuouse uma transformação dos dados para $\sqrt{ }$ x. Para as variáveis que apresentaram valor zero para algumas parcelas, como $\mathrm{N}^{\circ} \mathrm{G}$ e SEV, utilizou-se a transformação $\sqrt{x}+1 / 2$.

A significância dos efeitos de tratamentos foi avaliada pelo teste F. Para as características $\mathrm{N}^{\circ} \mathrm{G}$, SEV e FR, as médias foram comparadas pelo o teste $t$ $(\mathrm{P} \leq 0,05)$. Para essas mesmas características, efetuouse o teste correlação entre as médias avaliadas.

\section{RESULTADOS E DISCUSSÃO}

De acordo com os resultados obtidos na tabela 1, para todas as cultivares e linhagens de feijoeiro avaliadas, não foi observado nenhum material com resistência ao $\boldsymbol{M}$. javanica, uma vez que todos apresentaram fator de reprodução FR $>1$. Porém, existem diferenças significativas entre eles, e as cultivares com maior FR foram as 'Xamego' e 'Pérola', que apresentaram valores de 1,48 e 1,91, respectivamente, seguidas da 'cultivar' 'IAPAR 14', com FR=2,09. Dentre as linhagens com menor FR, destaca-se LP 9985.

Para a severidade ao $\boldsymbol{F}$. oxysporum f. sp. phaseoli, avaliada aos 35 e 60 dias da inoculação, foi observada diferença para as cultivares 'Pérola' e 'IAPAR 14', as quais apresentaram a menor SEV (nota um), seguidas da linhagem LP 9985, que se comportou como resistente (nota zero) nas duas épocas avaliadas.

Nas avaliações das inoculações simultâneas com os dois patógenos, os menores FR foram observados nas cultivares 'IPR Graúna' 'Xamego' e 'Pérola’ e na linhagem LP 9812, as quais apresentaram respectivamente os valores (notas) de 1,44; 1,76; 1,87; e 2,1 (Tabela 1). Com exceção da cultivar 'Xamego', para as demais foi observada redução no FR em comparação com os valores obtidos somente com a inoculação de $\boldsymbol{M}$. javanica.

Para a SEV de F. oxysporum f. sp. Phaseoli, foram observados acréscimos quando da interação entre a inoculação do nematoide, principalmente na avaliação aos 60 dias, para as cultivares 'IPR Graúna’, 'IPR Uirapuru’ e ‘Xamego’ e a linhagem LP98 123’. 
Tabela 1 - Reação de cultivares e linhagens de feijoeiro inoculado com M. javanica e Fusarium oxysporum f. sp. phaseoli, quanto ao número de Galhas (NG), Fator de Reprodução (FR) e severidade (SEV), avaliadas aos 35 e 60 dias.

\begin{tabular}{|c|c|c|c|c|c|c|c|c|c|}
\hline \multirow{2}{*}{ Cultivares/linhagens } & & \multicolumn{2}{|c|}{ Testemunha } & \multicolumn{2}{|c|}{ M. javanica } & \multicolumn{2}{|c|}{ Fofsp } & \multicolumn{2}{|c|}{$M j+F o f s p$} \\
\hline & & 35 & 60 & 35 & 60 & 35 & 60 & 35 & 60 \\
\hline \multirow[t]{3}{*}{ 'IAPAR 81’ } & NG & - & - & $65 a$ & - & - & - & $67 a$ & - \\
\hline & FR & - & - & - & $13,8 \mathrm{a}$ & - & - & - & $17,6 \mathrm{a}$ \\
\hline & SEV & - & - & - & - & За & $2 \mathrm{a}$ & За & За \\
\hline \multirow[t]{3}{*}{ ‘IPR Graúna’ } & NG & - & - & $31,3 b$ & - & - & - & $41,6 a$ & - \\
\hline & FR & - & - & - & $2,94 b$ & - & - & - & $1,44 a$ \\
\hline & SEV & - & - & - & - & $2 \mathrm{a}$ & За & За & $4 b$ \\
\hline \multirow[t]{3}{*}{ ‘Pérola’ } & NG & - & - & $70,1 \mathrm{a}$ & - & - & - & $67,5 a$ & - \\
\hline & FR & - & - & - & 1,91a & - & - & - & $1,87 a$ \\
\hline & SEV & - & - & - & - & $1 \mathrm{a}$ & $1 b$ & $1 \mathrm{a}$ & За \\
\hline \multirow[t]{3}{*}{ ‘IPR Uirapuru’ } & NG & - & - & $61 \mathrm{a}$ & - & - & - & $64 a$ & - \\
\hline & FR & - & - & - & $13,5 a$ & - & - & - & $11,1 \mathrm{a}$ \\
\hline & SEV & - & - & - & - & $2 \mathrm{a}$ & За & $3 b$ & $4 \mathrm{~b}$ \\
\hline \multirow[t]{3}{*}{ 'IAPAR 14’ } & NG & - & - & $34,8 \mathrm{a}$ & - & - & - & $40,0 \mathrm{a}$ & - \\
\hline & FR & - & - & - & 2,09a & - & - & - & $2,94 a$ \\
\hline & SEV & - & - & - & - & 0a & 1a & $1 \mathrm{a}$ & $2 b$ \\
\hline \multirow[t]{3}{*}{ 'Xamego' } & NG & - & - & $40,0 \mathrm{~b}$ & - & - & - & 32,8a & - \\
\hline & FR & - & - & - & $1,48 \mathrm{a}$ & - & - & - & $1,76 \mathrm{a}$ \\
\hline & SEV & - & - & - & - & За & За & За & $4 \mathrm{~b}$ \\
\hline \multirow[t]{3}{*}{ 'Rubi' } & NG & - & - & $26 a$ & - & - & - & $22 a$ & - \\
\hline & FR & - & - & - & $3,0 \mathrm{a}$ & - & - & - & $7,96 \mathrm{a}$ \\
\hline & SEV & - & - & - & - & За & За & 3a & $4 a$ \\
\hline \multirow[t]{3}{*}{ 'IPR Juriti' } & NG & - & - & $46 \mathrm{~b}$ & - & - & - & 30 & - \\
\hline & FR & - & - & - & $3,26 a$ & - & - & - & $4,02 \mathrm{a}$ \\
\hline & SEV & - & - & - & - & За & $4 a$ & $2 \mathrm{a}$ & За \\
\hline \multirow[t]{3}{*}{ 'IPR Chopin’ } & NG & - & - & $34 a$ & - & - & - & $34 a$ & - \\
\hline & FR & - & - & - & $4,1 \mathrm{a}$ & - & - & - & $3,7 \mathrm{a}$ \\
\hline & SEV & - & - & - & - & $2 \mathrm{a}$ & $2 \mathrm{a}$ & $2 \mathrm{a}$ & За \\
\hline \multirow[t]{3}{*}{ 'LP98 123’ } & NG & - & - & $34 a$ & - & - & - & 30a & - \\
\hline & FR & - & - & - & $2,94 a$ & - & - & - & $2,1 \mathrm{a}$ \\
\hline & SEV & - & - & - & - & $2 a$ & $4 a$ & $2 \mathrm{a}$ & $4 a$ \\
\hline \multirow[t]{3}{*}{ ‘LP99 85’ } & NG & - & - & $22 \mathrm{a}$ & - & - & - & $22 a$ & - \\
\hline & FR & - & - & - & $1,55 a$ & - & - & - & $2,46 a$ \\
\hline & SEV & - & - & - & - & $0 \mathrm{a}$ & $0 \mathrm{a}$ & $1 \mathrm{a}$ & $1 b$ \\
\hline
\end{tabular}

*Médias seguidas de mesma letra minúscula não diferem entre si na coluna pelo teste de Tukey, a 5\% de probabilidade.

A suscetibilidade de genótipos de feijoeiro ao parasitismo de nematoides do gênero Meloigogyne também foi observada por diferentes autores. SILVA \&
CAMPOS (2002) avaliaram a reação de 39 linhagens de feijoeiro e observaram que todos os materiais avaliados foram bons hospedeiros dos nematoides. Os autores 
observaram também que houve diferença de parasitismo entre os nematoides, mostrando que um mesmo genótipo de feijoeiro pode reagir de forma diferenciada frente às diferentes espécies de nematoides.

Os resultados obtidos no presente trabalho estão de acordo com os resultados relatados por SIMÃO et al. (2003), que, ao avaliarem o efeito de diferentes concentrações de inóculos de $\boldsymbol{M}$. javanica no desenvolvimento das cultivares de feijoeiro 'IAPAR 81' e 'Pérola', no período do verão e inverno, não observaram comportamento para resistência em nenhuma das cultivares avaliadas, observando que as cultivares de feijoeiro 'Pérola' e 'Iapar 81' comportaramse como altamente suscetíveis mesmo nas menores concentrações de inóculo de $\boldsymbol{M}$. javanica. Esses feijoeiros permitiram a reprodução de $\boldsymbol{M}$. javanica em suas raízes, nas diferentes concentrações e nos diferentes períodos avaliados.

LORDELLO et al. (1994), MOURA \& MOURA (1994) e REGAZZI et al. (1992) também não encontraram fontes de resistência a $\boldsymbol{M}$. javanica nos genótipos de feijoeiro avaliados. Entretanto, WALBER et al. (2003), ao avaliarem 54 cultivares de feijoeiro, encontraram fontes de resistência aos nematoides $\boldsymbol{M}$. javanica e $\boldsymbol{M}$. incognita nas cultivares 'Nemasnap' e 'Manoa Wonder', confirmando os resultados obtidos por CARNEIRO \& FERRAZ (1992).

A suscetibilidade ou resistência de plantas aos patógenos depende da interação entre hospedeiro $\mathrm{x}$ patógeno ou patógeno $\mathrm{x}$ patógeno. No presente estudo, ficou evidente a alteração do comportamento dos genótipos, principalmente da cultivar 'Pérola', em relação à murcha de Fusarium. Quando Fusarium oxysporum f. sp. phaseoli foi associado a M. javanica, ocorreu um aumento significativo da severidade da doença. A influência da interação entre nematoides do gênero Meloidogyne e Fusarium também foi observada por RIBEIRO \& FERRAZ (1983), os quais, ao avaliarem cinco genótipos de feijoeiro, constataram que, nos tratamentos em que havia o parasitismo simultâneo, ocorreu um aumento significativo na severidade da doença. Em outras espécies, tal fato também foi observado. Em algodoeiro, RUANO (1983) observou que a interação entre $\boldsymbol{M}$. javanica, $\boldsymbol{M}$. incognita raça 3 e $\boldsymbol{F}$. oxysporum f. sp. vasinfectum levou à ocorrência de maiores prejuízos à cultura em razão do ataque simultâneo dos patógenos.

Na tabela 1, verifica-se que a característica SEV a Fusarium oxysporum f. sp. phaseoli apresentou alta e significativa correlação com as características NG, FR e SEV. Esses resultados reforçam a evidência de que os sintomas de severidade a $\boldsymbol{F}$. oxysporum, nos genótipos de feijoeiro avaliados, estão diretamente relacionados à presença de fitonematoides, interferindo diretamente no desenvolvimento das plantas.

\section{CONCLUSÕES}

Nenhuma fonte de resistência a $\boldsymbol{M}$.javanica foi detectada neste estudo, uma vez que todas as cultivares e linhagens de feijoeiro avaliadas comportaram-se como suscetíveis.

Para Fusarium oxysporum f. sp. phaseoli isoladamente, as cultivares 'Iapar 14' e 'Pérola' e a linhagem LP99-85 apresentam-se como resistentes. Porém, este estudo evidencia a influência da interação entre o Fusarium oxysporum f. sp. phaseoli e o nematoide Meloidogyne javanica no aumento da severidade dos sintomas da murcha de Fusarium, levando à quebra da resistência na cultivar 'Pérola' .

\section{REFERÊNCIAS}

AGUDELO, F.V. Nematodes. In: SCHWARTZ, H.F.; GALVEZ, G.E. Bean production problems: disease insect, soil and climatic constraints of Phaseolus vulgaris. Cali: CIAT, 1980. p.315-326.

ATKINSON, G.F. Some disease of cotton. Alabama: Agricultural Experiment Station of the Agriculrural and Mediamical College, 1892. 65p.

BONETTI, J.I.S; FERRAZ, S. Modificações do método de Hussey e Barker para extração de ovos de Meloidogyne exigua em raízes de cafeeiro. Fitopatologia Brasileira, v.6, n.3, p.533, 1981

BOOKBINDER, M.G.; BLOOM, J.R. Interaction of Uromyces phaseoli and Meloidogyne incognita on bean. Journal of Nematology, v.12, p.177-182, 1980. Disponível em: <http:/ /fulltext10.fcla.edu/DLData/SN/SN0022300X/0012_003/ 80_30.pdf>. Acesso em: 21 dez. 2009.

COSTA, J.F. et al. Métodos de inoculação em plantas de feijoeiro. Fitopatologia Brasileira, v.19, n.2, p.125-128, 1989.

CRUZ, J.C. et al. Murcha de Fusarium em Feijoeiro: método de inoculação, reação de germoplasma e principais métodos de controle. Summa Phytopathologica, v.2, n.2, p.105-111, 1974.

HUSSEY, R.S.; BARKER R.K. Methods of collecting inoculation of Meloidogyne spp. inclusing a new technique. Plant Disease Reporter, v.57, p.1025-1028, 1973.

JULIATTI, F.C.; RUANO, O. Algodão (Gossipium hirsutum L.). Controle de doenças. Doenças causadas por fungos e bactérias. In: VALE, F.X.R.; ZAMBOLIM, L. Controle de doenças de plantas. Grandes culturas. Viçosa: Departamento de Fitopatologia, UFV; Brasília, Distrito Federal: Ministério da agricultura e do abastecimento, 1997. V.2, p.555-570. 
LORDELLO, A.A.L. et al. Estudos da interação entre Meloidogyne javanica e Fusarium oxysporum f. sp. phaseoli em feijoeiro comum. Nematologia Brasileira, v.8, p.77-81, 1994.

MOURA, R.M.; MOURA, A.M. Comportamentos de genótipos de Phaseolus vulgaris em relação aos nematoides Meloidogyne incognita raça 1 a M. javanica. Nematologia Brasileira, v.18, p.50-56, 1994.

MOURA, R.M.; REGIS, E.M.O. Reação de cultivares de feijoeiro comum (Phaseolus vulgaris) em relação ao parasitismo de Meloidogyne javanica e $\boldsymbol{M}$. incognita (Nematoda: Heteroderidae). Nematologia Brasileira, v.11, p.215-225, 1987

OOSTENBRINK, R. Major characteristics of the relation between nematodes and plants. Mededelingen der Landbouwhogeschool, v.66, n.3, p.1-46, 1966.

PEDROSA, E.M.R. et al. Resposta de genótipos de Phaseolus vulgaris à meloidoginoses e alguns mecanismos envolvidos na reação. Fitopatologia Brasileira, v.25, n.2, p.190-196, 2000.

REGAZZI, A.J. et al. Estudos de mecanismos de resistência a Meloidogyne incognita, raça 3, em variedades de feijoeiro. Nematologia Brasileira, v.16, n.1/2, p.41-52, 1992.

RIBEIRO, C.A.G.; FERRAZ, S. Estudos da interação entre Meloidogyne javanica e Fusarium oxysporum f. sp. phaseoli. Fitopatologia Brasileira, v.8, n.3, p.439-446, 1983.

ROBERTS, P.A. Current status of the availability, development, and use of host plant resistance to nematodes. Journal of Nematology, v.24, p.213-217, 1992. Disponível em: <http:/ /www.ncbi.nlm.nih.gov/pmc/articles/PMC2619273/pdf/ 213.pdf>. Acesso em: 21 dez. 2009.
RUANO, O. Resistência de algodoeiro (Gossypium spp.) à Meloidogyne incognita (Kofoid \& White) Chitwood raça 3 e Fusarium oxysporum Schelecht f. sp. vasinfectum (Atk) Snyder \& Hansen e à associação desses organismos. 1984. 38f. Dissertação (Mestrado em Fitopatologia) - Curso de Pósgraduação em Agronomia, Universidade Federal de Viçosa, MG.

SAS INSTITUTE - STATISTICAL ANALYSIS SYSTEM. SAS/ STAT $^{\circledR}$ user' guide. Version 6 . The GLM procedure. 4.ed. Cary, 1990. V.2, cap.24, p.891-996.

SILVA. J.V.; CAMPOS, H.D. Reação de cultivares de feijão a nematoides de galhas. In: CONGRESSO NACIONAL DE PESQUISA DE FEIJÃO, 7., 2002, Viçosa, MG. Resumos expandidos... Viçosa: Universidade Federal de Viçosa, 2002. p.102.

SIMÃO, G. et al. Níveis de inóculo de Meloidogyne javanica no desenvolvimento de feijoeiro cultivado no verão. In: MOSTRA ACADÊMICA DE TRABALHOS DE AGRONOMIA - MATA, 7., 2003, Londrina, PR. Resumo... Londrina: Universidade Estadual de Londrina, 2003. p.126.

SINGH, D.B. et al. Effect of root-knot nematode Meloidogyne incognita on Fusarium wilt of French beans. Indian Journal of Nematology, v.11, p.84-85, 1981. Disponível em: <http://www.indianjournals.com/ ijor.aspx?target $=$ ijor:ijn\&volume $=11 \&$ issue $=1 \&$ article $=022>$. Acesso em: 21 dez. 2009.

VIEIRA, C. Doenças e pragas do feijoeiro. Viçosa: UFV, 1993. 231p.

WALBER, R. et al.. Avaliação de acessos de feijoeiro em relação aos nematoides das galhas. In: CONGRESSO BRASILEIRO DE FITOPATOLOGIA, 36., 2003, Uberlândia, MG. Resumo... Uberlândia: Fitopatologia Brasileira, 2003. V.28, p.293-294. 